\title{
THE CONVERSE OF THE FATOU THEOREM FOR POSITIVE HARMONIC FUNCTIONS
}

\author{
BY
}

LYNN H. LOOMIS

1. Introduction. Let $v(z)=v\left(r e^{i \phi}\right)$ be a function harmonic in the unit circle $|z|<1$ and admitting there the Poisson-Stieltjes representation

$$
v\left(r e^{i \phi}\right)=\frac{1}{2 \pi} \int_{0}^{2 \pi} \frac{1-r^{2}}{1+r^{2}-2 r \cos (\theta-\phi)} d V(\theta),
$$

where $V(\theta)$ is of bounded variation over $0 \leqq \theta \leqq 2 \pi$. The Fatou theorem( $\left.{ }^{1}\right)$, in one form, has the following to say about the relation between $v(z)$ and $V(\theta)$ in (1):

Theorem A. If $V_{(1)}(\theta)=\lim _{t \rightarrow 0}(V(\theta+t)-V(\theta-t)) / 2 t$ exists, then $V\left(r e^{i \theta}\right)$ $\rightarrow V_{(1)}(\theta)$ as $r \rightarrow 1$.

Theorem B: If the derivative $V^{\prime}(\theta)$ exists, then $v(z) \rightarrow V^{\prime}(\theta)$ as $z \rightarrow e^{i \theta}$ along any chord of $|z|<1$ (hence along any "non-tangential path" or "in angle").

The converses of these theorems are in general not true. If $v(z)$ is positive however, both converses can be proved. One result is that if $v\left(r e^{i \theta}\right)$ is a bounded function harmonic in $|z|<1$, and if its boundary function $v(\theta)$ is defined as the limit, wherever it exists, of $v(z)$ as $z \rightarrow e^{i \theta}$ "in angle," then $v(\theta)$ is a summable function which is precisely equal to the derivative of its indefinite integral. The converse of Theorem A for positive functions follows readily from known theorems, and it is the main object of this paper to deduce from it a strengthened form of the converse of Theorem $B$ for positive functions.

We shall have occasion to use the theorem $\left({ }^{2}\right)$ that a harmonic function has the representation (1) if and only if it can be written as the difference of two non-negative (or two positive) harmonic functions. In particular, every positive harmonic function has the representation (1) with $V(\theta)$ increasing.

2. The converse of Theorem A for positive functions. It will be simplest to infer the converse of Theorem A for positive functions from a series of remarks.

(i) The limit (if it exists) $V_{(1)}(\theta)=\lim _{t \rightarrow 0}[V(\theta+t)-V(\theta-t)] / 2 t$ is known as the generalized symmetric derivative of $V(\theta)$.

Presented to the Society, May 3, 1941 under the title $A$ converse to the Fatou theorem; received by the editors May 12, 1942.

(1) Fatou's original paper is in the Acta Math. vol. 30 (1906) pp. 335-400.

(2) See Evans, Logarithmic potential. Discontinuous Dirichlet and Neumann problems, Amer. Math. Soc. Colloquium Publications vol. 6, 1927, p. 48. 
(ii) If $\sum\left(a_{n} \cos n \theta+b_{n} \sin n \theta\right)$ is the Fourier-Stieltjes series for $d V(\theta)$, then $v\left(r^{i \theta}\right)=\sum r^{n}\left(a_{n} \cos n \theta+b_{n} \sin n \theta\right)$ is the Fourier series expansion for $v(z)$, so that the existence of the $\operatorname{limit} \lim _{r \rightarrow 1} v\left(r e^{i \theta}\right)$ is equivalent (by definition) to the Abel summability (summability A) of the series $\sum\left(a_{n} \cos n \theta+b_{n} \sin n \theta\right)$.

(iii) It is a well known theorem $\left(^{3}\right)$ that a series is summable $(C, n+1)$ if it is summable $\mathrm{A}$ and if its $n$th Cesàro means are positive.

(iv) It is elementary that the $(C, 1)$ means of the Fourier-Stieltjes series of a non-decreasing function are positive $\left({ }^{4}\right)$.

(v) By a theorem of Hardy and Littlewood $\left.{ }^{5}\right)$, summability $(C, \alpha)$ with $\alpha>0$ for the Fourier-Stieltjes series of a non-decreasing function implies summability $(C, \beta)$ for every $\beta>0$, and is equivalent to the existence of the generalized symmetric derivative $V_{(1)}(\theta)$.

The converse of Theorem A for positive functions follows directly from these remarks.

3. The Poisson-Stieltjes integral in the half-plane. For some purposes it is convenient to work with integral representations in the half-plane rather than in the circle. A function $u(x, y)$ harmonic in the half-plane $y>0$ admits the Poisson-Stieltjes integral representation

$$
u(x, y)=\frac{1}{\pi} \int_{-\infty}^{\infty} \frac{y\left(1+t^{2}\right)}{y^{2}+(t-x)^{2}} d U(t),
$$

where $U(t)$ is of bounded variation over the closed infinite interval $[-\infty, \infty]$, if and only if the transformed function $v(w)$ obtained by mapping the halfplane (by $z=i(1-w) /(1+w)$ ) on to the unit.circle $|w|<1$ has the PoissonStieltjes representation (1), where $U(\tan \theta / 2)=V(\theta) / 2$. Note that (2) is not actually an improper integral, for the integrand is continuous over the closed infinite interval $[-\infty, \infty]$ and $U(t)$ is of bounded variation there; also note that $U(t)$ may have a jump at infinity. Obviously $U^{\prime}(t)$ $=V^{\prime}(2 \arctan t) /\left(1+t^{2}\right)$ when either derivative exists; thus $U^{\prime}(0)_{i}=V^{\prime}(0)$. We can rewrite (2) by removing the jump of $U(t)$ at infinity as $k y$ and writing $U_{1}(t)=\int_{0}^{t}\left(1+t^{2}\right) d U(t)$. Then (2) becomes

$$
u(x, y)=k y+\frac{1}{\pi} \int_{-\infty}^{\infty} \frac{y}{y^{2}+(t-x)^{2}} d U_{1}(t) .
$$

The integral is absolutely convergent and the kernel is simpler than the kernel of (2). Also $U_{1}^{\prime}(t)=V^{\prime}(2 \operatorname{arc} \tan t)$ so that the Fatou theorem is generally valid. On the other hand, $U_{1}(t)$ is not of bounded variation in the in-

(3) See Kogbetliantz, Sommation des séries et intégrales divergentes par les moyennes arithmétiques et typique, Mémorial des Sciences Mathématiques vol. 51 p. 40, Theorem 21.

(4) See Titchmarsh, Theory of functions, p. 412. It is only necessary to replace the Lebesgue integral by a Stieljes integral in the equation for $\sigma_{n}$.

(5) See Zygmund, Trigonometrical series, p. 263 and p. 266, Example 11. 
finite interval. We shall find it convenient to use (2) rather than (3), and adjust the mapping of $|w|<1$ onto $y>0$ so that any desired boundary point maps to the origin $z=0$ where the desired Fatou relation holds.

If $U_{1}(t)$ is absolutely continuous with derivative $u(t)$ then (3) becomes

$$
u(x, y)=k y+\frac{1}{\pi} \int_{-\infty}^{\infty} \frac{y}{y^{2}+(t-x)^{2}} u(t) d t .
$$

If the original function $v(w)$ admits the ordinary Poisson representation

$$
v\left(r e^{i \phi}\right)=\frac{1}{2 \pi} \int_{0}^{2 \pi} \frac{1-r^{2}}{1+r^{2}-2 r \cos (\theta-\phi)} v(\theta) d \theta,
$$

the transformed function has the representation (4) with $k=0$ and $u(t)$ $=v(2 \arctan t)$.

For the purposes of this paper, the factor $1+t^{2}$ in the numerator of the integrand of (2) may be dropped. We are interested in limiting behavior as $z$ approaches the origin along rays $(r x, r y), 0<r \leqq 1, y>0$. But

$$
\int_{-\infty}^{\infty} \frac{(r y) t^{2}}{(r y)^{2}+(t-r x)^{2}} d U(t)=r \int_{-\infty}^{\infty} \frac{y t^{2}}{y^{2}+(t-x)^{2}} d U(r t) .
$$

We consider an angle space by restricting $x$ to $-x_{0}<x<x_{0}$. The integrand of the right member of (6) is bounded over $-x_{0}<x<x_{0},-\infty \leqq t \leqq \infty$, so that the absolute value of the integral is bounded by $r M V$ (where $V$ is the variation of $U(t))$. Thus this term approaches 0 uniformly as $z$ approaches the origin in any angle space, and we can disregard it. We have left to consider the harmonic function, again denoted $u(x, y)$,

$$
u(x, y)=\frac{1}{\pi} \int_{-\infty}^{\infty} \frac{y}{y^{2}+(t-x)^{2}} d U(t)
$$

which can be written

$$
u(r x, r y)=\frac{1}{\pi} \int_{-\infty}^{\infty} \frac{y}{y^{2}+(t-x)^{2}} d \frac{U(r t)}{r} .
$$

The Fatou Theorems A and B follow at once from (8). The assumption of Theorem $\mathrm{A}$ is that $[U(t)-U(-t)] / 2 t \rightarrow U_{(1)}(0)$ as $t \rightarrow 0$. Thus $[U(r t)$ $-U(-r t)] / r=2 t(U)_{(1)}(0)+t R(r t)$ where $|R(t)|$ is bounded, say by $M$, and $|R(t)| \rightarrow 0$ as $t \rightarrow 0$. For $x=0$, (8) becomes

$$
u(0, r y)=\frac{1}{\pi} \int_{0}^{\infty} \frac{y}{y^{2}+t^{2}} d\left[\frac{U(r t)-U(-r t)}{r}\right],
$$

and if we substitute the above expression in (9) and integrate the last term by parts, we have 


$$
\begin{aligned}
u(0, r y) & =U_{(1)}(0)+\frac{2}{\pi} \int_{0}^{\infty} R(r t) \frac{t^{2} y}{\left(y^{2}+t^{2}\right)^{2}} d t \\
& =U_{(1)}(0)+\frac{2}{\pi} \int_{0}^{\pi / 2} R(r t) \sin ^{2} \beta d \beta,
\end{aligned}
$$

where $\beta=\arctan t / y$. For $r$ small enough, $|R(r t)|$ is arbitrarily small over as large a part of the range of $t$, and hence of $\beta$, as desired. Over the remaining part of the range of $\beta$ the integrand is bounded by $M$. Therefore the integral approaches 0 , and $U_{(1)}(0)=\lim _{r \rightarrow 1} u(0, r y)$ which is the conclusion of Theorem A.

Theorem B can as easily be inferred. We assume $U(0)=0$, and have $U(r t) / r=t U^{\prime}(0)+t R(r t)$, and instead of (10) this gives

$$
u(r x, r y)=U^{\prime}(0)+\frac{2}{\pi} \int_{-\infty}^{\infty} R(r t) \frac{t(t-x) y}{\left[y^{2}+(t-x)^{2}\right]^{2}} d t .
$$

Now $\left|t(t-x) /\left(y^{2}+(t-x)^{2}\right)\right|<K$ over $-x_{0}<x<x_{0},-\infty \leqq t \leqq \infty$, and the absolute value of the integral is therefore bounded by

$$
\frac{2 K}{\pi} \int_{-\pi / 2}^{\pi / 2}|R(r t)| d \beta
$$

which approaches 0 with $r$ as in the proof of Theorem A.

If one will compare these proofs with the corresponding proofs carried out in the unit $\operatorname{circle}\left({ }^{6}\right)$, the advantages of the half-plane representations will be appreciated.

Using the representation (7) the converse of Theorem A for positive functions can be deduced immediately from the following integral Tauberian theorem of Hardy and Littlewood $\left({ }^{7}\right)$.

Theorem. Let $f(t)$ be positive, and suppose that $f(t) /(t+x)^{p} \in L(0, \infty)$ for some (and so for all) $x>0$. Suppose that

$$
\int_{0}^{\infty} \frac{f(t)}{(t+x)^{\rho}} d t \sim \frac{H}{x^{\sigma}}
$$

as $x \rightarrow \infty($ as $x \rightarrow 0)$ for $0<\sigma<\rho$. Then

as $t \rightarrow \infty$ (as $t \rightarrow 0)$.

$$
F(t)=\int_{0}^{t} f(u) d u \sim \frac{H \Gamma(\rho)}{\Gamma(\sigma) \Gamma(\rho-\sigma+1)} t^{\rho-\sigma}
$$

The statement of the theorem can be modified to include Stieltjes integra-

(') See Evans, loc. cit., pp. 39-43.

(7) Hardy and Littlewood, On Tauberian theorems, Proc. London Math. Soc. (2) vol. 30 (1930) p. 25. 
tion, and then only elementary changes of variable are required to put the theorem in a form directly applicable to the Poisson integral for the half-plane.

We shall have occasion to use the following theorem $\left({ }^{8}\right)$.

Theorem. Let $U(t)$ have a jump $m$ at $t=0$; thus $m=0$ is equivalent to the continuity of $U(t)$ at $t=0$. Then as $z=x+i y$ approaches the origin along the ray $x=R y, y u(x, y)$ approaches the value $m /\left(1+k^{2}\right) \pi$. In particular, $U(t)$ is continuous at $t=0$ if and only if $y u(x, y)$ approaches 0 along some ray (and hence along all rays).

In proof we consider

$$
(r y) u(r x, r y)=\frac{1}{\pi} \int_{-\infty}^{\infty} \frac{y^{2}}{y^{2}+(t-x)^{2}} d U(r t) .
$$

Now as $r \rightarrow 0, U(r t) \rightarrow V(t)$ where $V(t)=U(0+)$ for $0<t<\infty, V(t)=U(0-)$ for $-\infty<t<0$, and $V( \pm \infty)=U( \pm \infty)$. Then by the Helly-Bray theorem concerning the convergence of sequences of Stieltjes integrals $\left({ }^{9}\right)$,

$$
(r y) u(r x, r y) \rightarrow \frac{1}{\pi} \int_{-\infty}^{\infty} \frac{y^{2}}{y^{2}+(t-x)^{2}} d V(t)=\frac{m y^{2}}{\pi\left(y^{2}+x^{2}\right)} .
$$

Since $x=k y$ the theorem follows.

4. The converse of Theorem B for positive functions. We shall prove the stronger theorem:

THEOREM 1. Let $u(z)=u(x, y)$ be a positive harmonic function in the upper half-plane $y>0$, and having therefore the representation (2) with $U(t)$ increasing. If $\lim u(z)=u(0)$ as $z$ approaches the origin along each of two rays, than $U^{\prime}(0)$ exists and equals $u(0)$. (2).

We shall carry through the proof in a number of steps, using (7) instead of

(i) If $u(z) \rightarrow u(0)$ as $z \rightarrow 0$ along each of two rays then $u(z) \rightarrow u(0)$ uniformly as $z \rightarrow 0$ between the rays. If the angle space between the rays is opened up to a half-plane by a power $w=e^{i \beta} z^{\alpha}$ we obtain from $u(z)$ a new positive harmonic function $u_{1}(x, y)$ continuous in the closed half-plane $y \geqq 0$ except possibly at the origin, and having a boundary function $u_{1}(t)$ which is continuous at the origin if it is defined there to have the value $u(0)$. Since $u_{1}(x, y)$ is positive it admits the representation (7) where $U_{1}(t)$ has the continuous derivative $u_{1}(t)$ when $t \neq 0$. Thus $U_{1}(t)$ is absolutely continuous if

(8) Fejér, Über die Bestimmung des Sprunges der Funktion aus ihrer Fourierreihe, J. reine angew. Math. vol. 142 (1913) pp. 165-166. See also Warschawski, Bemerkung zu meiner Arbeit: Über das Randverhalten der Ableitung der Abbildungsfunktion bei konformer Abbildung, Math. Zeit. vol. 38 (1934) p. 682.

( ${ }^{\circ}$ See D. V. Widder, The Laplace transform, p. 31. 
it has no jump discontinuity at the origin. But by the theorem at the end of the last section, $U(t)$ is continuous at $t=0$, which implies successively that $r u(r x, r y) \rightarrow 0$ as $r \rightarrow 0$ for every point $(x, y)$ in the upper half-plane $y>0$, that similarly $r u_{1}(r x, r y) \rightarrow 0$, and finally that $U_{1}(t)$ is continuous at the origin. Therefore $U_{1}(t)$ is absolutely continuous and $u_{1}(x, y)$ has the representation

$$
u_{1}(x, y)=\frac{1}{\pi} \int_{-\infty}^{\infty} \frac{y}{y^{2}+(t-x)^{2}} u_{1}(t) d t .
$$

The continuity of $u_{1}(t)$ at the origin implies the continuity of $u_{1}(x, y)$ at the origin, which proves the assertion (i).

(ii) Next, $u(z) \rightarrow u(0)$ as $z \rightarrow 0$ along any ray to the origin. Let $l_{1}$ and $l_{2}$ be the two given rays and suppose that $l_{2}$ makes the positive angle $\alpha$ with $l_{1}$. Let $l_{3}$ make the angle $\alpha$ with $l_{2}$ (we suppose that $l_{3}$ lies in the upper half-plane). We shall show that if $l:(r x, r y)$ is any ray between $l_{2}$ and $l_{3}$, then $u(r x, r y)$ $\rightarrow u(0)$ as $r \rightarrow 0$. We need only to open up the angle space between $l_{1}$ and $l_{3}$ as in (i); $l_{2}$ becomes the ray perpendicular to the axis at the origin. The new positive harmonic function $u_{1}(x, y)$ has the representation (2) and by hypothesis (a) $u_{1}(0, r y) \rightarrow u(0)$ as $r \rightarrow 0$, (b) $U_{1}(t)$ is absolutely continuous for $t>0$, and $u_{1}(t)=U_{1}^{\prime}(t) \rightarrow u(0)$ as $t \rightarrow 0$ (from the right). Thus $U_{1}(t) / t \rightarrow u(0)$ as $t \rightarrow 0$ from the right. Application of the converse of Theorem A for positive functions shows that $U_{1}(t) / t \rightarrow u(0)$ as $t \rightarrow 0$ from the left. Thus $U_{1}^{\prime}(0)$ exists and equals $u(0)$. By the Fatou theorem $u_{1}(r x, r y) \rightarrow u(0)$ as $r \rightarrow 0$ for every $(x, y)$ with $y>0$. This proves the statement about rays between $l_{2}$ and $l_{3}$. The assertion (i) together with a finite number of applications of the above process, proves (ii).

(iii) It remains to prove from these facts that $U^{\prime}(0)$ exists and equals $u(0)$. By the converse of Theorem A it is sufficient to prove that $U(t) / t \rightarrow u(0)$ as $t \rightarrow 0$ from the right. The obvious device is to open up an angle space having the positive real axis as one of its bounding rays. But it is then somewhat difficult to establish the relation between the functions $U_{1}(t)$ and $U(t)$ for positive $t$. We shall proceed differently. Integrating (7) by parts we have

$$
u(x, y)=\frac{1}{\pi} \int_{-\infty}^{\infty} \frac{2 y(t-x)}{\left[y^{2}+(t-x)^{2}\right]^{2}} U(t) d t .
$$

By (ii)

$$
u(0, r y)=\frac{1}{\pi} \int_{-\infty}^{\infty} \frac{2 t y}{\left(y^{2}+t^{2}\right)^{2}} \frac{U(r t)}{r} d t \rightarrow u(0) \quad \text { as } r \rightarrow 0 .
$$

We can assume that $U(0)=0$, and the integrand in (12) is accordingly nonnegative. We can therefore integrate and invert the order of integration, giving 


$$
\begin{aligned}
\int_{0}^{1} u(0, r y) d r & =\frac{1}{\pi} \int_{-\infty}^{\infty} \frac{2 t y}{\left(y^{2}+t^{2}\right)^{2}}\left(\int_{0}^{1} \frac{U(r t)}{r} d r\right) d t \\
& =\frac{1}{\pi} \int_{-\infty}^{\infty} \frac{2 t y}{\left(y^{2}+t^{2}\right)^{2}}\left(\int_{0}^{t} \frac{U(r)}{r} d r\right) d t
\end{aligned}
$$

In particular, $U(r) / r$ is integrable over every finite interval. We can now perform the same operation on (11), justifying the change in the order of integration by absolute integrability. Thus

$$
u_{1}(x, y)=\int_{0}^{1} u(r x, r y) d r=\frac{1}{\pi} \int_{-\infty}^{\infty} \frac{2 y(t-x)}{\left[y^{2}+(t-x)^{2}\right]}\left(\int_{0}^{t} \frac{U(r)}{r} d r\right) d t,
$$

and integrating by parts,

$$
u_{1}(x, y)=\frac{1}{\pi} \int_{-\infty}^{\infty} \frac{y}{y^{2}+(t-x)^{2}} \frac{U(t)}{t} d t .
$$

The function $u_{1}(z)=u_{1}(x, y)$ is obviously harmonic and positive in the upper half-plane, and $u_{1}(z) \rightarrow u(0)$ as $z \rightarrow 0$ along any ray $(r x, r y), y>0$. We now employ the device suggested at the beginning of (iii). Let $l_{1}$ and $l_{2}$ make angles $\alpha(\alpha<\pi / 2)$ and $2 \alpha$ with the positive real axis, and apply the transformation $w=z^{\pi / 2 \alpha}$. The harmonic function $u_{1}(z)$ has the boundary function $u_{1}(t)=U(t) / t$. After the transformation, the new harmonic function $u_{2}(w)$ has the boundary function $u_{2}(t)=u_{1}\left(t^{2 \alpha / \pi}\right)$. In the Stieltjes form $U_{2}^{\prime}(t)=u_{2}(t)$ and by definition $U_{2}(t) / t \rightarrow u(0)$ as $t \rightarrow 0$ from the left. By the converse of Theorem A for positive functions

$$
\frac{U_{2}(t)}{t}=\frac{1}{t} \int_{0}^{t} u_{2}(s) d s \rightarrow u(0)
$$

as $t \rightarrow 0$ from the right. The following lemma is due to Landau $\left({ }^{10}\right)$.

If $x f^{\prime}(x)$ increases with $x$ and $f(x) \sim x^{a}(a>0)$ as $x \rightarrow 0$, then $f^{\prime}(x) \sim a x^{a-1}$ as $x \rightarrow 0$.

Here

$$
f(t)=\int_{0}^{t} u_{2}(s) d s \sim t u(0)
$$

as $t \rightarrow 0$, and $t f^{\prime}(t)=t u_{2}(t)=t^{1-2 \alpha / \pi} U\left(t^{2 \alpha / \pi}\right)$ which increases with $t$. Therefore by Landau's lemma, $u_{2}(t) \sim u(0)$ as $t \rightarrow 0$, that is, $U(t) / t \rightarrow u(0)$ as $t \rightarrow 0$ from the right. We now apply the converse of Theorem A again to obtain $U(t) / t \rightarrow u(0)$ as $t \rightarrow 0$ from the left. Thus $U^{\prime}(0)$ exists and equals $u(0)$, and the proof of the theorem is complete.

(10) E. Landau, Beitrage zur analytischen Zahlentheorie, Rend. Circ. Mat. Palermo vol. 24 (1917) pp. 81-160. 
It should be remarked that the direct converse of Theorem $\mathbf{B}$ for positive functions can be proved from considerations of the integral representation (7) without any reference to the converse of Theorem A.

5. A counterexample. In this section we shall show by a counterexample that neither of the converses of A and B is true for the general representation (2). We first define the function $U(t)$ and then define the harmonic function $u(z)$ by the representation (7). The graph of $U(t)$ will consist of a sequence of triangular peaks separated by intervals of the $t$-axis and converging to the origin, the vertices of the peaks lying on the line $s=t$ over the points $t=2^{-n}$, the slopes of the sides of the peaks to be determined by later considerations. Such a function $U(t)$ is clearly of bounded variation.

We thus define $U(t)$ as follows:

$$
U(t)= \begin{cases}0, & t<0 \\ 0, & t>1 \\ (1 / 2)^{n}, & t=(1 / 2)^{n}, n=1,2, \cdots \\ 0, & t=(1 / 2)^{n} \pm a_{n}, 0 \leqq a_{n} \leqq 2^{-n-3},\end{cases}
$$

$U(t)$ linear on $2^{-n}-a_{n} \leqq t \leqq 2^{-n}$ and on $2^{-n} \leqq t \leqq 2^{-n}+a_{n}$, and $U(t)=0$ elsewhere in $0<t<1$. The $a_{n}$ are positive numbers to be chosen later subject to the restriction noted above. On $2^{-n}-a_{n}<t<2^{-n}, d U(t)=2^{-n} d t / a_{n}$ so that

$$
\begin{aligned}
u(x, y) & =\frac{1}{\pi} \int_{-\infty}^{\infty} \frac{y}{y^{2}+(t-x)^{2}} d U(t) \\
& =\frac{1}{\pi} \sum_{n=1}^{\infty}\left[\int_{2^{-n}-a_{n}}^{2^{-n}} \frac{1}{2^{n} a_{n}} \frac{y}{y^{2}+(t-x)^{2}} d t\right. \\
& =\frac{1}{\pi} \sum_{n=1}^{\infty} \int_{2^{-n}}^{2^{-n+a_{n}}} \frac{1}{2^{n} a_{n}}\left[\frac{y}{y^{2}+\left(t-x-a_{n}\right)^{2}}-\frac{1}{y^{2}+(t-x)^{2}}\right] d t \\
& \left.=\frac{1}{\pi} \sum_{n=1}^{\infty} \int_{2^{-n}}^{2^{-n}+a_{n}} \frac{y}{2^{n}} \frac{y}{\left[y^{2}+(t-x)^{2}\right.} d t\right]
\end{aligned}
$$

Thus

$$
|u(x, y)| \leqq \frac{1}{\pi} \sum_{n=1}^{\infty} \frac{a_{n}}{2^{n}} \cdot \max _{2^{-n} \leqq t \leqq 2^{-n+a_{n}}}\left|\frac{y\left[2(t-x)-a_{n}\right]}{\left[y^{2}+\left(t-x-a_{n}\right)^{2}\right]\left[y^{2}+(t-x)^{2}\right]}\right| .
$$

Consider the term

$$
\left|\frac{y}{2} \frac{2(t-x)-a_{1}}{\left[y^{2}+\left(t-x-a_{1}\right)^{2}\right]\left[y^{2}+(t-x)^{2}\right]}\right|
$$


on a ray $x=k y$. If we allow $x, y, t$ and $a_{1}$ to vary, subject to the restrictions $0 \leqq a_{1} \leqq 1 / 8,1 / 2-a_{1} \leqq t \leqq 1 / 2+a_{1}, x=k y$, the term has a maximum value $M_{k}$. By homogeneity the general term

$$
\left|\frac{y}{2^{n}} \frac{2(t-x)-a_{n}}{\left[y^{2}+\left(t-x-a_{n}\right)^{2}\right]\left[y^{2}+(t-x)^{2}\right]}\right|
$$

with $0 \leqq a_{n} \leqq 2^{-n-3}, 2^{-n}-a_{n} \leqq t \leqq 2^{-n}+a_{n}, x=k y$ has the maximum value $2^{n} M_{k}$. Now choose the constants $a_{n}$ as $2^{-2 n}$. Then $a_{n}$ times the general term above is bounded by $2^{-n} M_{k}$.

It is clear that the general term approaches 0 as $y$ approaches $0(x=k y)$ uniformly over the allowed range of $t$. Given $\epsilon$, choose $N$ so that

$$
\sum_{n=N+1}^{\infty} 2^{-n} M_{k}<\epsilon / 2
$$

and choose $y_{0}$ so that for $y<y_{0}$ and $x=k y$, the sum of the first $N$ terms is bounded in absolute value by $\epsilon / 2$. Thus for $y<y_{0}$ and $x=k y,|u(x, y)|<\epsilon$, and we have proved that $u(x, y) \rightarrow 0$ as $z \rightarrow 0$ along any ray to the origin. It is obvious however that $U(t) / t$ oscillates between 0 and 1 as $t \rightarrow 0$ from the right, and that $[U(t)-U(-t)] / 2 t$ oscillates between 0 and $1 / 2$ as $t \rightarrow 0$ from the right.

6. Generalizations and applications. The procedure of Theorem 1 is adequate for situations more general than that described there. Suppose, for instance, we have not that $u(x, y)$ approaches $u(0)$ but that

$$
\int_{0}^{1} u(r x, r y) d r
$$

exists and approaches $u(0)$ as $z=x+i y$ approaches the origin along each of two rays $l_{1}$ and $l_{2}$ We open up the angle space as before and get a positive harmonic function $u_{1}(x, y)$ admitting the representation

with the hypothesis that

$$
u_{1}(x, y)=\frac{1}{\pi} \int_{-\infty}^{\infty} \frac{y}{y^{2}+(t-x)^{2}} u_{1}(t) d t
$$

$$
\int_{0}^{1} u_{1}\left( \pm|r t|^{\beta}\right) d r \rightarrow u(0)
$$

as $r \rightarrow 0$, two separate statements being understood. Here $\beta=\pi / \alpha$ where $\alpha$ is the angle between $l_{1}$ and $l_{2}$. We now need the following lemma:

LemMa. If $u(t)$ is a positive function such that for some $\alpha>0$

$$
\int_{0}^{0} u\left(t^{\alpha}\right) d t \sim k s
$$


as $s \rightarrow 0$, then

$$
\int_{0}^{s} u(t) d t \sim k s
$$

In proof we multiply both sides by $s^{\alpha-2}$ and integrate from 0 to $r$. If we then integrate the left member by parts the conclusion follows.

If $r t$ is replaced by $t$ in (13) and the lemma applied, we obtain as a conclusion precisely the hypothesis of the Fatou Theorem B for the harmonic function $u_{1}(x, y)$ (with $U(s)$ absolutely continuous and equal to the integral (14)). Therefore $u_{1}(z) \rightarrow u(0)$ as $z \rightarrow 0$ "in angle," which is equivalent to the statement that $u(z) \rightarrow u(0)$ as $z \rightarrow 0$ along any path between $l_{1}$ and $l_{2}$. We can therefore apply Theorem 1 to infer that $U^{\prime}(0)$ exists and equals $u(0)$. Also

$$
\int_{0}^{1} u(s x, s y) d s=\frac{1}{r} \int_{0}^{r} u(t \cos \theta, t \sin \theta) d t
$$

where $z=x+i y=r(\cos \theta+i \sin \theta)$, and the new hypothesis is thus that the integral Hölder mean approaches $u(0)$. We have thus proved the following theorem:

THEOREM 2. Let $u(z)=u(x, y)$ be a positive harmonic function in the upper half-plane $y>0$, and having therefore the representation (2) with $U(t)$ increasing. If $u(z)$ has the $(H, 1)$ limit $u(0)$ as $z=x+i y$ approaches the origin along each of two rays, then $U^{\prime}(0)$ exists and equals $u(0)$.

CoRollary 1. As a consequence of the Fatou theorem $u(x, y)$ has the ordinary limit $u(0)$ as $z=x+i y$ approaches the origin along any ray of the upper halfplane.

COROllary 2. If $u(z)$ has the $(H, n)$ limit $u(0)$ as $z$ approaches the origin along each of two rays, then $U^{\prime}(0)$ exists and equals $u(0)$.

This is a trivial consequence of the Landau lemma used in $\$ 4$, for if $u(t)$ is positive, and

$$
\int_{0}^{s} \frac{1}{t} \int_{0}^{t} u(r) d r \sim s u(0)
$$$$
s \rightarrow 0,
$$

then direct application of the lemma gives that

$$
\int_{0}^{t} u(r) d r \sim t u(0)
$$

A finite number of such steps reduces the hypothesis of the corollary to that of Theorem 2.

Again, it is clear that we have used the monotonicity of $U(t)$ (positivity 
of $u(x, y))$ only locally about the origin, and the hypothesis can be accordingly weakened to that extent.

It is interesting to see what can be said for other statements of the Fatou theorem. A somewhat stronger form of the theorem than that contained in the first section concerns the ordinary Poisson integral representation

$$
v\left(r e^{i \phi}\right)=\frac{1}{2 \pi} \int_{0}^{2 \pi} \frac{1-r^{2}}{1+r^{2}-2 r \cos (\theta-\phi)} v(\theta) d \theta .
$$

Let $v_{\theta}(z)=\partial v\left(r e^{i \theta}\right) / \partial \theta$. Then $v_{\theta}(z)$ is a harmonic function in $|z|<1$ which does not in general admit a Poisson-Stieltjes integral representation.

THEOREM $\mathrm{A}^{\prime}$. If $v_{(1)}(\theta)=\lim _{t \rightarrow 0}[v(\theta+t)-v(\theta-t)] / 2 t$ exists, then $v_{\theta}\left(r e^{i \theta}\right)$ $\rightarrow v_{(1)}(\theta)$ as $r \rightarrow 1$.

Theorem $\mathrm{B}^{\prime}$. If the derivative $v^{\prime}(\theta)$ exists, then $v_{\theta}(z) \rightarrow v^{\prime}(\theta)$ uniformly as $z \rightarrow e^{i \theta}$ "in angle."

The proofs are essentially the same as those for Theorems A and B. As before, we must impose some further restriction on $v(\theta)$ in order to deduce the converses of Theorems $\mathrm{A}^{\prime}$ and $\mathrm{B}^{\prime}$, and we try the local condition that, $v(\theta)-v\left(\theta_{0}\right)$ change sign at $\theta_{0}$ that is, that $\left[v(\theta)-v\left(\theta_{0}\right)\right]\left(\theta-\theta_{0}\right)$ be of constant sign (admitting the value 0 ) in some neighborhood of $\theta_{0}$. We may obviously take $v\left(\theta_{0}\right)=0$. Thus in the half-plane our hypotheses are that $u(x, y)$ has the representation

$$
u(x, y)=\frac{1}{\pi} \int_{-\infty}^{\infty} \frac{y}{y^{2}+(t-x)^{2}} u(t) d t,
$$

where the integral is absolutely convergent, that $t u(t) \geqq 0$ in some neighborhood of the origin, and the $\partial u(x, y) / \partial x=u_{x}(x, y)$ has the property that $u_{x}(r x, r y) \rightarrow l$ as $r \rightarrow 0$ for every $(x, y)$ with $y>0$. For the converse of $\mathrm{A}$, the assumption holds only along the ray $x=0$. Now

$$
u_{x}(x, y)=\frac{1}{\pi} \int_{-\infty}^{\infty} \frac{2 y(t-x)}{\left[y^{2}+(t-x)^{2}\right]} u(t) d t,
$$

and

$$
u_{x}(0, r y)=\frac{1}{\pi} \int_{-\infty}^{\infty} \frac{2 y t}{\left[y^{2}+t^{2}\right]^{2}} \frac{u(r t)}{r} d t .
$$

The integrand here is positive in the neighborhood of $r=0, t=0$, and since $u_{x}(0, r y)$ is integrable over $0<r<1$ we have

$$
\int_{0}^{1} u_{x}(0, r y) d r=\frac{1}{\pi} \int_{-\infty}^{\infty} \frac{2 y t}{\left[y^{2}+t^{2}\right]^{2}}\left(\int_{0}^{t} \frac{u(r)}{r} d r\right) d t
$$

and in general 


$$
\begin{aligned}
\int_{0}^{1} u_{x}(r x, r y) d r & =\frac{1}{\pi} \int_{-\infty}^{\infty} \frac{2 y(t-x)}{\left[y^{2}+(t-x)^{2}\right]}\left(\int_{0}^{t} \frac{u(r)}{r} d r\right) d t \\
& =\frac{1}{\pi} \int_{-\infty}^{\infty} \frac{y}{y^{2}+(t-x)^{2}} \frac{u(t)}{t} d t
\end{aligned}
$$

Since $u(t) / t$ is non-negative in some neighborhood of $t=0$, we can apply Theorem 1 with $u(t) / t=U^{\prime}(t)$ to obtain

$$
\frac{1}{t} \int_{0}^{t} \frac{u(r)-u(-r)}{2 r} d r \rightarrow l
$$

as $t \rightarrow 0$, as the converse of Theorem $\mathrm{A}^{\prime}$, and

$$
\frac{1}{t} \int_{0}^{t} \frac{u(r)}{r} d r \rightarrow l
$$

as $t \rightarrow 0$, as the converse of Theorem $\mathrm{B}^{\prime}$.

To obtain the symmetry of the earlier case we should now prove that (a) and (b) can be taken as weakened hypotheses for the Fatou Theorems $A^{\prime}$ and $B^{\prime}$. This is in fact the case, but we shall omit the proofs here since they are essentially the same as the proofs of Theorems A and B.

The relation between $v_{\theta}(w)$ in the unit circle and $u_{x}(x, y)$ in the half-plane can be easily established. They are different functions even when transformed so as to have the same domain of definition, but they have the same asymptotic properties at the origin in the half-plane.

If $f(z)$ is a bounded analytic function in the unit circle $|z|<1$ it is, known $\left({ }^{11}\right)$ that if $\lim f(z)$ exists as $z$ approaches a boundary point $e^{i \theta}$ along some curve, then $f(z)$ has that limit as $z \rightarrow e^{i \theta}$ "in angle." Thus there is no difference between situations $A$ and $B$ in this case. The Fatou theorem implies $\left({ }^{12}\right)$ that $\lim _{r \rightarrow 1} f\left(r e^{i \theta}\right)$ exists for almost all $\theta$, and the converse of the Fatou theorem (Theorem 1) implies that if $f(\theta)$ is the boundary function thus defined then $f(\theta)$ is precisely equal to the derivative of its indefinite integral.

(11) See Nevanlinna, Eindeutige Analytische Funktionen, p. 65.

(12) See Bieberbach, Funktionentheorie, II, pp. 147-148.

HARVARD UNIVERSITY,

Cambridge, Mass. 